\title{
The Game Equilibrium of Scientific Crowdsourcing Solvers Based on the Hotelling Model
}

\author{
Guohao Wang and Liying Yu *D \\ School of Management, Shanghai University, Shanghai 200444, China; guohaowang@shu.edu.cn \\ * Correspondence: yuliying@shu.edu.cn; Tel.: +86-021-66133851
}

Received: 12 August 2019; Accepted: 24 October 2019; Published: 29 October 2019

check for updates

\begin{abstract}
Scientific crowdsourcing, which can effectively obtain wisdom from solvers, has become a new type of open innovation to address worldwide scientific and research problems. In the crowdsourcing process, the initiator should satisfy his own research needs by selecting a proper solver from the crowd, and the solver must have multiple competitions in order to obtain scientific research tasks from the initiator. The participants in the scientific crowdsourcing are based on the knowledge flow to realize the value added of knowledge. This paper discusses a few factors, including knowledge utility, knowledge transfer cost, knowledge distance, and knowledge trading cost, which all affect the solvers to achieve game equilibrium and win tasks in scientific crowdsourcing. By referring to the concept of Hotelling model, this paper constructs a game model with the solvers as the participants, and analyses solvers' behaviours in scientific crowdsourcing and their profit impacts by each of the key elements. The results show that from a crowdsourcing solver's point of view, increasing knowledge utility, controlling knowledge transfer cost, shortening knowledge distance to the initiator, and leveraging with a knowledge trading cost are four effective approaches to wining the competition of a scientific crowdsourcing task. The research conclusions provide a theoretical basis and practice guidance for crowdsourcing solvers to participate in scientific crowdsourcing from the perspective of the knowledge flow process.
\end{abstract}

Keywords: scientific crowdsourcing; game model; knowledge flow; equilibrium

\section{Introduction}

As initiated by Jeff Howe [1], crowdsourcing, a type of "open innovation," refers to an effort to leverage the expertise of a global pool of individuals and organizations, to as quickly and cost effectively as possible develop and implement creative solutions to innovation challenges. Across the world, previous studies on crowdsourcing have mainly focused on one or several of the following: collaboration with the crowd [2], ideal implementation impacted by crowd experience [3], making use of crowdsourcing to create new products, collecting distributed data, solving problems more effectively [4,5], improving organizational decision making [6], or improving organizational openness to external new knowledge [7,8]. In particular, in terms of evaluating the importance of crowdsourcing for organizational learning, it is considered to be a "new, exciting direction of research" [9-11]. Nowadays, in order to improve organizations' innovation abilities, some scholars [12-14] started to investigate the knowledge flow process in scientific crowdsourcing. Crowdsourcing, from the relationship between the initiator and the solver, is only a process of issuing tasks and completing tasks, and this is essentially a process of knowledge flow. Only by realizing the flow of knowledge can the crowdsourcing relationship become sustainable.

To the author's knowledge, the current primary domain of research has not touched the base of the equilibrium for scientific crowdsourcing solution solvers, especially in a quantitative approach when participating in scientific crowdsourcing activities. In the process of scientific crowdsourcing, 
the initiator has the resources and funds, while the solver has the creativity and technology. Both parties need to work together to improve the quality of knowledge transfer and make maximum profit. On the one hand, when knowledge flows from solver to initiator, the initiator gets a benefit while there are costs to the solver; on the other hand, once the solve wins scientific crowdsourcing tasks, profit will be achieved. Thus, it is very important to understand the balance of knowledge flow and profit. That way, the knowledge flow decision-making behaviour of the crowdsourcing subjects can be understood as the process of a game between the initiator and the solver [15]. This study used the Hotelling game model to study scientific crowdsourcing activities' equilibrium, which is basically a Nash Equilibrium, and used the mechanism with two crowdsourcing solvers. More specifically, this paper constructs a game model to investigate how solvers participate in the competition to win the task, from a knowledge flow perspective. This paper aims to:

1. Introduce the game model into the scientific crowdsourcing equilibrium decision process and analyse solvers' behaviours;

2. Simulate a knowledge flow process based on scientific crowdsourcing with four key elements, which are knowledge utility, knowledge transfer cost, knowledge distance, and knowledge trading cost;

3. Analyse how these key elements affect a solver's profit in the scientific crowdsourcing process.

The rest of the paper is organized as follows: recent literature is reviewed in Section 2. Model assumptions, notation, and formulations are developed in Sections 3 and 4. Numerical simulations and discussions are presented in Section 5. Finally, some conclusive remarks are provided in Section 6.

\section{Literature Review}

Scientific crowdsourcing is a new type of research cooperation mode. Initiators, who are usually research institutions or scientists, aim to scientifically discover and solve the challenges of technology. The rise of scientific crowdsourcing is based on two key elements: Internet as a platform [16] and knowledge as a flowing medium [17], which means, that knowledge flows from crowdsourcing solver to crowdsourcing initiator in a solid transferring process via the Internet. In the scientific crowdsourcing process, knowledge, the only good to sell and purchase between solver and initiator, can bring monetary reward to the solver and knowledge utility to initiator, while a crowdsourcing solver is strongly driven by either monetary incentive or nonmonetary gain, or both. As for nonmonetary gain [18], it mainly includes social recognition, self-esteem, new knowledge acquisition, individual skill development, etc. From the focal firm's point of view, gaining knowledge from an individual or organization is one of the most important motivations [19].

In scientific crowdsourcing activities, the knowledge flow process can be described as the exchange and utilization of knowledge, which often needs to be integrated through external collaborations [20]. Knowledge integration is critical to the competitiveness and the survival of a firm, especially for an emerging market [21]. On the Innocentive website, one of the largest crowdsourcing platforms for innovation problems, enterprises post challenging tasks, which are usually new product development or new technique-related, in the form of scientific crowdsourcing [22]. According to the research of Malhotra et al. [23], it is easier to create a smooth knowledge flow process for an internal crowd than external crowd participants. Wang et al. [24] analysed knowledge transfer decision-making behaviour for the initiator and solver in scientific crowdsourcing. Fang et al. [25] made use of crowdsourcing to resolve unlabelled data for modelling creation. An organizational network's information transformation, R\&D collaboration, and a firm's acquisition of knowledge flow play key roles that ensure technological innovation. It is obvious that firms can obtain knowledge utility, including solutions, ideas, data, experience, information, etc., during scientific crowdsourcing. Xue et al. [26] have shown that in the knowledge transfer process, the knowledge collaboration effect, knowledge transfer cost, and knowledge transfer potential are three key factors impacting knowledge transfer behaviour. 
According to Albors et al. [27], crowdsourcing enables the diffusion of learning and access to knowledge; it is usually determined by the processes of solver's knowledge dissemination and the initiator's knowledge absorption, including knowledge identification, assimilation, internalization, and application [28]. Since different organizations have knowledge that is not easily transmitted across boundaries, previous researches found that articulated and codified knowledge can improve crowdsourcing effectiveness [29,30]. Naidorf [31] found that knowledge mobilization, which includes push and pull of knowledge between individuals and groups for mutual benefit, is a way of making knowledge useful and increasing the value of knowledge's utility. Furthermore, knowledge's intrinsic characterizations, such as knowledge property, level of abstraction, tacit knowledge, etc. have great impacts on the success of the knowledge flow process [32,33]. Despite benefits from an external organization, the technique or knowledge transfer process generates a non-negligible cost, particularly in complex technological knowledge transfer [34]. Chen [35] introduces the notion of "distance of knowledge" to a simply three-dimensional model, which includes knowledge coding, abstraction extent, and the buyer's knowledge base. The "knowledge distance" would determine knowledge transfer cost and the deal be made when the buyer and seller reach to the shortest "knowledge distance." Capaldo et al. [36] think there are two kinds of knowledge distance, technological distance and geographical distance, which have opposite effect on value contribution. Piazza et al. [12] found that knowledge distances between initiators and solvers tend to act as complements in specific governance structure decisions. Galati et al. [37] developed an innovative model for knowledge transfer, including key factors like partners' knowledge similarities, etc. In our research, we use knowledge distance instead of knowledge similarities. A shorter knowledge distance means more knowledge similarities between the initiator and the solver.

In crowdsourcing, the motivation for participants varies a lot for different solvers. Some solvers are purely monetary motivated, and some others desire to obtain new knowledge or accumulate experience [38], and the achievements of new knowledge acquisition are very attractive to solvers. However, in order to motivate solvers intrinsically and/or extrinsically, to attract more effort from the solvers, crowdsourcing initiators could share some of their knowledge, such as experience, methods, techniques, information, etc., to external individuals or organizations, or even competitors, which may cause ineffective IP management [39]. Such knowledge spill-over from scientific crowdsourcing initiators may weaken their competitive and innovative capabilities [40]. In the research of Jiang et al. [41], regarding knowledge leakage, there is a "U-shaped pattern" in the knowledge flow within a competitive alliance. It is not unusual that solvers' motivations are nonmonetary incentives, such as knowledge spill-over from the initiator, to improve their own knowledge structures and innovative abilities.

Furthermore, due to the global Internet's development, scientific crowdsourcing tends to become a worldwide activity, which draws attention from multinational crowdsourcing solvers. The cross-country trading cost could be a very important factor during the internationalization of the knowledge flow process [42]. According to Cassiman et al. [43], there are three types of cost affecting the inbound and outbound knowledge flow, including cognitive cost, transactional cost, and organizational cost. During scientific crowdsourcing's knowledge flow, it is clearly necessary to consider the imperfections of external costs, such as tariffs on trade, restrictions on the flow of capital, information cost, multinational organization cost, etc. Our research takes knowledge trading cost as of one the key elements in the knowledge flow of scientific crowdsourcing.

The above studies were mainly focused on the characterization of scientific crowdsourcing and the knowledge flow process, crowdsourcing solvers' participation motivations, and different applications for scientific crowdsourcing. Although there are some researchers qualitatively studying knowledge flow in the scientific crowdsourcing process, it is rare to see quantitative analyses in such a domain. This paper builds an equilibrium analysis based on game model and quantitatively evaluate solvers' knowledge characteristics and behaviour by four key elements, which are knowledge utility, knowledge transfer cost, knowledge distance, and knowledge trading cost in scientific crowdsourcing. 
The main contribution of this paper should be practical enlightenment for understanding the scientific crowdsourcing process.

\section{Assumptions and Notation}

This part focuses on assumptions and notations of the knowledge flow process in scientific crowdsourcing. Based on this paper's research interest, it is necessary to analyse how these key elements, including knowledge utility, knowledge transfer cost, knowledge distance, and knowledge trading cost impact the final profits for both crowdsourcing solvers.

In the paper, we consider two different crowdsourcing solvers and one crowdsourcing initiator. Specifically, the crowdsourcing task is knowledge transfer between solvers and initiator. The two solvers, who are assumed monetary motivated, try to compete to win the task and maximize each profit by transferring their knowledge to the crowdsourcing initiator. The monetarily motivated solvers will make decisions based on profit they can get from scientific crowdsourcing, while our research will explore the nonmonetary impact on a solver's profit, so that we can provide some guidance beyond monetary considerations.

In the paper, it is assumed the two crowdsourcing solvers have different knowledge bases to the crowdsourcing initiator. Here, knowledge utilities are denoted by $g_{A}$ and $g_{B}$ and are defined as nonmonetary gain for solver A and solver B. Obviously, if $g_{A}=g_{B}$, it means that both the crowdsourcing solvers transfer the same knowledge utility. And if $g_{i}>g_{j}$, it implies that the $i$ th solver transferred the superior knowledge to the initiator. We assume that the knowledge is perfectly perceived by the crowdsourcing initiator.

The other dimension to consider is crowdsourcing solvers' knowledge distance to the initiator. Similar knowledge types and characterizations between solvers and initiators means small knowledge distance. To interpret it otherwise, knowledge distance implies the distribution of crowdsourcing initiator's preference for these two solvers in a linear knowledge space. The reason that the Hotelling game model was selected in this paper is that the Hotelling model is a classical and simplified space competition model, which has been already applied in many other domains, such as regional economics [44], industry economics [45], political elections [46], etc. In the knowledge space this paper raises, the distance between initiator and solver just fits the Hotelling game model. It is assumed that the knowledge length of solvers is at unity and each of them are located at the opposite end points; in particular, solver $\mathrm{A}$ is located at $x=0$ and solver $\mathrm{B}$ is located at $x=1$.

The crowdsourcing initiator's preference is assumed to be uniformly distributed over the length of the knowledge space. Then, the initiator at knowledge space $x$ faces a knowledge transfer cost of $c \times x$ when he absorbs knowledge from solver $\mathrm{A}$, and $c \times(1-x)$ when absorbs knowledge from solver B. Therefore, $c>0$ is the transfer cost per unit knowledge distance. It is further assumed that the knowledge brings the initiator the largest utility, which is derived by gross utility minus the transfer $\operatorname{cost} c^{*} x_{i}$ and knowledge spill-over loss $s_{i}$.

Based on above assumptions and notation, the solver's game model for knowledge flow in scientific crowdsourcing is shown in Figure 1. In this model, solver A and solver B are located at $(0,0)$ and $(1,0)$ accordingly, and the initiator's knowledge location is $(x, 0)$. The linear segment represents their knowledge space. During the scientific crowdsourcing knowledge flow process, the direction of knowledge utility flow is from solver to initiator, while the knowledge spill-over flow is in the reverse direction, which is from initiator to solver. The knowledge transfer cost, which is determined by unit transfer cost and the knowledge distance $x$, is also a key factor in the model. In reality, considering the solver's continuous learning ability, which leads to shorter knowledge distance from the initiator, this paper also analysed the profit impact when solvers' knowledge distance changed to new locations $\left(p_{A}, 0\right)$ and $\left(p_{B}, 0\right)$.

We should also be aware that the model proposed in this paper is only applicable for two solvers to participate in crowdsource research activities, which is another assumption in the research. Future 
research could explore other good approaches to model more than two solvers' equilibriums in scientific crowdsourcing.

As of now, in order to have good model understanding, it is necessary to summarize all parameters in a table which will be used in next sections, as shown in Table 1.

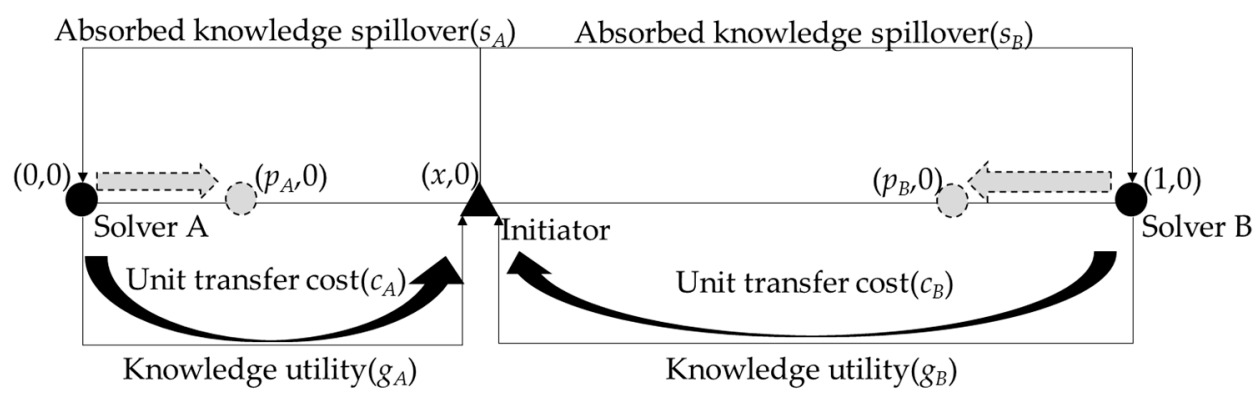

Figure 1. Knowledge flow in scientific crowdsourcing.

Table 1. Model parameter notation.

\begin{tabular}{cc}
\hline Parameter & Description \\
\hline$U_{0}$ & Maximum willingness for crowdsourcing initiator to pay \\
$g_{i}$ & Solver $i^{\prime}$ 's knowledge utility obtained by initiator \\
$c_{i}$ & Solver $i^{\prime}$ 's unit knowledge transfer cost \\
$x$ & Knitial knowledge distance between solver and initiator \\
$t$ & Knowledge trading cost \\
$h$ & Knowledge production cost \\
$s_{i}$ & Initiator's knowledge spill-over absorbed by solver $i$ \\
$\mu_{i}$ & Solver $i$ 's knowledge transfer cost coefficient \\
$p_{i}$ & Solver $i$ 's new knowledge location to initiator \\
$\pi_{i}$ & Solver $i^{\prime}$ 's knowledge profit \\
\hline
\end{tabular}

\section{Model Formulation}

\subsection{The Solver's Game Model under the Knowledge Flow in Scientific Crowdsourcing}

When a crowdsourcing initiator chooses a solver to start the knowledge transfer, the knowledge flow process will include both knowledge utility, which is from solver to initiator, and knowledge spill-over, which is from initiator to solver.

From the initiator's point of view, the following indirect utility expressions will be compared:

$$
U= \begin{cases}U_{0}+g_{A}-s_{A}-c_{A} x & \text { if transferring from solver } A \\ U_{0}+g_{B}-s_{B}-c_{B}(1-x) & \text { if transferring from solver } B\end{cases}
$$

where $U_{0}>0$ is the maximum willingness for a crowdsourcing initiator to pay and $U_{0}$ is assumed to be high enough to fully cover the knowledge transfer cost.

Somewhere along the knowledge distance's interval, a location $x_{0}$ exists, such that the crowdsourcing initiator faces indifference between choosing either solver's knowledge. The location $x_{0}$ is obtained by equating the indirect utilities above. Therefore, the knowledge transfer demands for solver $A$ and solver $B$ are, respectively:

$$
\begin{gathered}
x_{A}=x_{0}=\frac{c_{B}+g_{A}-g_{B}-s_{A}+s_{B}}{c_{A}+c_{B}} ; \\
x_{B}=1-x_{0}=\frac{c_{A}-g_{A}+g_{B}+s_{A}-s_{B}}{c_{A}+c_{B}},
\end{gathered}
$$


It is interesting to note that increasing solver $A^{\prime}$ s knowledge utility $g_{A}$ will increase solver $A^{\prime}$ s knowledge transfer demand, while it decreases the knowledge transfer demand for solver $B$. Since $s_{i}$ is the initiator's knowledge spill-over loss, it is also considered the solver's knowledge spill-over gain. Each solver's knowledge profit function is defined as:

$$
\pi_{i}=\left(s_{i}-h\right) x_{i}(i=A, B),
$$

where $h$ stands for knowledge producing cost. To maximize profit, we assume that each solver will simultaneously decide knowledge spill-over. Based on Equation (4), for solvers $A$ and $B$, the solution $\partial \pi_{i} / \partial s_{i}=0$ yields the following equilibrium spill-over gain result:

$$
\begin{aligned}
& s_{A}=h+\frac{c_{A}+2 c_{B}+g_{A}-g_{B}}{3} ; \\
& s_{B}=h+\frac{2 c_{A}+c_{B}-g_{A}+g_{B}}{3} .
\end{aligned}
$$

The $i^{\text {th }}$ solver spill-over gain is higher when $i^{t h}$ solver knowledge utility is higher, while it will be lower when $j^{\text {th }}$ solver knowledge utility is higher.

With Equations (2), (3), (5) and (6), knowledge transfer demand can be derived as follows:

$$
\begin{aligned}
& x_{A}=\frac{c_{A}+2 c_{B}+g_{A}-g_{B}}{3\left(c_{A}+c_{B}\right)} ; \\
& x_{B}=\frac{2 c_{A}+c_{B}-\left(g_{A}-g_{B}\right)}{3\left(c_{A}+c_{B}\right)} .
\end{aligned}
$$

With Equations (5)-(8), profits under separation are as follows:

$$
\begin{aligned}
& \pi_{A}=\frac{\left(c_{A}+2 c_{B}+g_{A}-g_{B}\right)^{2}}{9\left(c_{A}+c_{B}\right)} ; \\
& \pi_{B}=\frac{\left(2 c_{A}+c_{B}-g_{A}+g_{B}\right)^{2}}{9\left(c_{A}+c_{B}\right)} .
\end{aligned}
$$

The profit expressions from Equations (9) and (10) indicate that each solver's profit is basically related to the both solvers' knowledge utilities. The $i^{\text {th }}$ solver profit will increase when $i^{\text {th }}$ solver's knowledge utility increases, while the $i^{\text {th }}$ solver's profit will reduce when $j^{\text {th }}$ solver's knowledge utility increases. As a consequence, the competence level is clearly shown here between two crowdsourcing solvers.

Based on above modelling and discussion, the following proposition was derived.

Proposition 1. A crowdsourcing solver's knowledge utility has a positive impact on self-profit, while it has negative impact on their competitor's profit.

In scientific crowdsourcing activities, both solvers are in competition mode, and each solver's target is to have higher expected payoff than the other. The profit gap $\Delta \pi_{A}$ for solver $A$ is calculated by $\pi_{A}-\pi_{B}$. Based on Equations (9) and (10), $\Delta \pi_{A}$ is calculated as follows:

$$
\Delta \pi_{A}=\pi_{A}-\pi_{B}=\frac{\left(c_{A}+2 c_{B}+g_{A}-g_{B}\right)^{2}}{9\left(c_{A}+c_{B}\right)}-\frac{\left(2 c_{A}+c_{B}-g_{A}+g_{B}\right)^{2}}{9\left(c_{A}+c_{B}\right)} .
$$


The above Equation (11) can be solved and simplified as follows:

$$
\Delta \pi_{A}=\frac{2\left(g_{A}-g_{B}\right)-\left(c_{A}-c_{B}\right)}{3} .
$$

From solver $A$ 's point of view, in order to win the crowdsourcing task competition with solver $B$, it requires him to have higher expected profit, which means that $\Delta \pi_{A}>0$. From Equation (12), it is necessary to satisfy following inequality:

$$
g_{A}-g_{B}>\frac{c_{A}-c_{B}}{2}
$$

Similarly, the inequality formula can also be derived for solver $B$.

From Equation (13), this inequality formula indicates that, in order to have higher expectation of profit in crowdsourcing task competition, solver $i$ should make the gap of knowledge utility be larger than the half of the knowledge transfer cost gap, compared with solver $j$. In other words, from crowdsourcing initiator's point of view, the best choice is to select solver who has lower transfer cost and higher knowledge utility, which, in practical terms, usually means that solver needs to improve his knowledge value, e.g., the quality of solutions, ideas, data, experience, information, etc., and optimize his internal operation process to achieve lower knowledge transfer cost.

The above result can be written formally.

Proposition 2. In order to gain higher profit than a competitor and eventually win a task from by crowdsourcing initiator, a crowdsourcing solver should make sure the gap of knowledge utility is larger than the half gap of the knowledge transfer cost.

\subsection{What is the Impact from Knowledge Distance between Solver and Initiator?}

In reality, solvers usually have an internal learning process during the crowdsourcing activity, so that both of them can dynamically update related knowledge distance to each other. As mentioned, it is a competition process when both solvers participate in crowdsourcing task. This subsection investigates the impact of solvers' knowledge distance in crowdsourcing activity, which is how solvers come out with the best profit and equilibrium when both solvers' related knowledge distance changes.

For a knowledge space interval, it is assumed that both solvers' knowledge transfer costs are the same, and that each solver's knowledge has changed to location $p_{A}$ and $p_{B}$, both of which are still within range of $0-1$, as shown in Figure 1 . In addition, $p_{A}$ and $p_{B}$ locate at two sides of $x_{0}$ accordingly, and $p_{A}$ is at the left side of $p_{B}$; that means the inequation $0<p_{A}<x_{0}<p_{B}<1$. The knowledge distance between crowdsourcing initiator, who is at knowledge location $x$, and solvers are respectively derived as $x-p_{A}$ and $p_{B}-x$. Along this line of consideration, the knowledge transfer cost is $c^{*}\left(x-p_{A}\right)$ when the initiator absorbs knowledge from solver $\mathrm{A}$ and $c^{*}\left(p_{B}-x\right)$ when absorbs knowledge from solver $\mathrm{B}$.

Consequently, the updated indirect utility expressions are as follows:

$$
U= \begin{cases}U_{0}+g_{A}-s_{A}-c\left(x-p_{A}\right) & \text { if transferring from solver } A \\ U_{0}+g_{B}-s_{B}-c\left(p_{B}-x\right) & \text { if transferring from solver } B\end{cases}
$$

The knowledge transfer demand, knowledge spill-over and profit are derived as below:

$$
\begin{gathered}
x_{A}=\frac{\left[g_{A}-g_{B}+\left(1+p_{A}\right) c+\left(1+p_{B}\right) c\right]}{6 c} ; \\
x_{B}=\frac{\left[g_{B}-g_{A}+\left(2-p_{A}\right) c+\left(2-p_{B}\right) c\right]}{6 c} ;
\end{gathered}
$$




$$
\begin{gathered}
s_{A}=\frac{1}{3}\left[g_{A}-g_{B}+\left(1+p_{A}\right) c+\left(1+p_{B}\right) c\right]+h ; \\
s_{B}=\frac{1}{3}\left[g_{B}-g_{A}+\left(2-p_{A}\right) c+\left(2-p_{B}\right) c\right]+h ; \\
\pi_{A}=\frac{\left[g_{A}-g_{B}+\left(1+p_{A}\right) c+\left(1+p_{B}\right) c\right]^{2}}{18 c} ; \\
\pi_{B}=\frac{\left[g_{B}-g_{A}+\left(2-p_{A}\right) c+\left(2-p_{B}\right) c\right]^{2}}{18 c} .
\end{gathered}
$$

From Equation (19), solver $A$ 's profit is positively impacted by the values of $p_{A}$ and $p_{B}$. With the definition of $p_{A}$ and $p_{B}$ and based on the research model in Figure 1, an increasing $p_{A}$ value means shortening the knowledge distance between solver $A$ and the initiator, while increasing the $p_{B}$ value means adding knowledge distance between solver $B$ and the initiator. In a similar observation from Equation (20), solver $B^{\prime}$ s profit is negatively impacted by the values of $p_{A}$ and $p_{B}$. A decreasing $p_{A}$ value means adding knowledge distance between solver $A$ and initiator, while a decreasing $p_{B}$ value means shortening the knowledge distance between solver $B$ and the initiator.

To sum up, when solver i's location is closer to initiator's knowledge location, solver $i$ 's profit will increase, and when solver $i$ 's location is farther to initiator's knowledge location, solver $i$ 's profit will decrease. The practical implication is that in crowdsourcing, the faster learner could potentially be the better solver, and thus gain more profit than other competitors.

Hence, Proposition 3 is written down below.

Proposition 3. Knowledge distance between the solver and initiator has a meaningful impact on crowdsourcing profit. A smaller knowledge distance would come with higher profit expectations for the solver.

\subsection{What Is the Solver's Game Equilibrium When Considering Knowledge Flow across Countries?}

Let us consider the situation that there are two scientific crowdsourcing solvers and one of them is in different country to the initiator. Assume solver $A$ is a local participant, the same nationality as the initiator, and solver $B$ is a foreign participant. Furthermore, assume that there is a trading cost $(t)$ to be incurred when knowledge flows between the initiator and the foreign solver. Obviously, if $t$ $=0$, the distinction between foreign and local solver becomes blurred, and in that case the model in Section 4.1 is still valid. With $t>0$, the utility function can be rewritten as follows:

$$
U= \begin{cases}U_{0}+g_{A}-s_{A}-c x & \text { if transferring from solver } A \\ U_{0}+g_{B}-s_{B}-c(1-x)-t & \text { if transferring from solver } B\end{cases}
$$

By using the same calculation method in Section 4.1, the knowledge transfer demand and profit can be derived as follows:

$$
\begin{aligned}
& x_{A}=\frac{1}{6 c}\left(3 c+g_{A}-g_{B}+t\right) ; \\
& x_{B}=\frac{1}{6 c}\left(3 c-g_{A}+g_{B}-t\right) ; \\
& s_{A}=\frac{g_{A}-g_{B}+t}{3}+c+h ; \\
& s_{B}=\frac{g_{B}-g_{A}-t}{3}+c+h ;
\end{aligned}
$$




$$
\begin{aligned}
& \pi_{A}=\frac{\left(3 c+g_{A}-g_{B}+t\right)^{2}}{18 c} ; \\
& \pi_{B}=\frac{\left(3 c-g_{A}+g_{B}-t\right)^{2}}{18 c} .
\end{aligned}
$$

By comparing Equations (26) and (27), the payoffs gap $\Delta \pi$ is calculated as follows:

$$
\Delta \pi=\pi_{A}-\pi_{B}=\frac{2}{3}\left(g_{A}-g_{B}+t\right) .
$$

For solver $A$, in order to win competition, the $\Delta \pi$ should be larger than 0 , as shown follows:

$$
t>g_{B}-g_{A}
$$

Similarly, the inequality formula can also be derived for solver $B$.

From Equation (29), when the trading cost $t$ is larger than $g_{j}-g_{i}$, the crowdsourcing solver i will always expect to get higher profit than foreign solver $j$, even if solver i's knowledge utility is less than solver $j$ 's. In this case, trading cost $t$ is leveraging on the competition. It can be formally written down as follows:

Proposition 4. Given a positive trading cost, even if the local crowdsourcing solver has lower knowledge utility than the foreign solver, the local solver will still win the competition if the trading cost is sufficiently large.

\section{Numerical Simulation and Discussion}

In the following sections, some numerical simulation examples, with presumptions of some parameters' values, are presented to analyse key factors influencing knowledge flows in scientific crowdsourcing and the solver's equilibrium. The factors presented here are knowledge utility $g_{i}$, knowledge transfer cost $u_{i}^{*} c$, knowledge distance $p_{i}$, and knowledge trading cost $t$.

\subsection{Knowledge Utility and Transfer Cost}

As a number of the aforementioned studies illustrated that nonmonetary factors, such as utility from new knowledge learning, are some of the key motivations for crowdsourcing a solver's participation. In order to explore the impact of knowledge utility on a crowdsourcing solver's utility, it is natural to analyse a basic model with the assumption of $c_{A}=c_{B}=1$. The numerical simulation for both solvers' profits versus their knowledge utilities are shown in Figure 2.

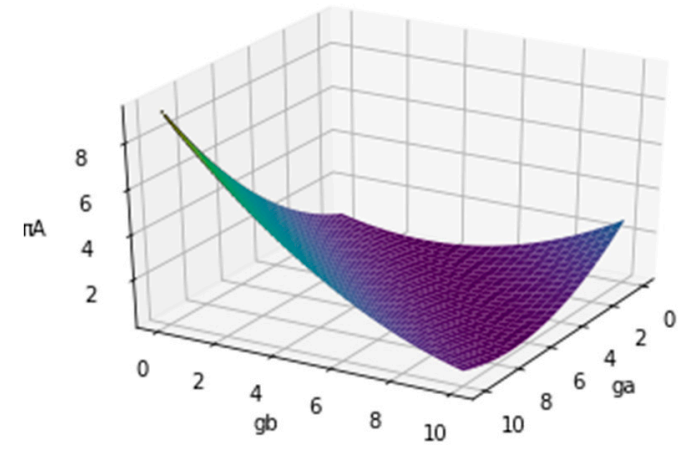

(a)

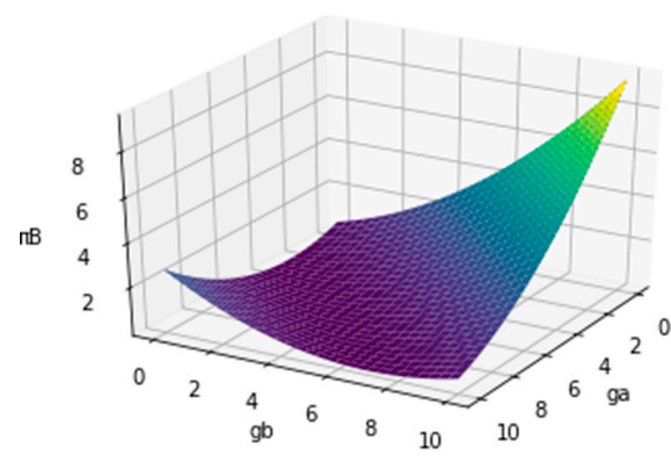

(b)

Figure 2. (a) solver- $A$ 's profit versus knowledge utility; (b) solver- $B$ 's profit versus knowledge utility.

The simulation results clearly show the competition status between two solvers during the crowdsourcing knowledge flow process. Basically, the profit of solver $i$ will increase when $i$ 's 
knowledge utility increases, while the profit of solver $i$ will decrease when the competitor, solver $j$ 's knowledge utility increases. For solver $i$, the most profitable point appears when solver $i$ has the largest knowledge utility while solver $j$ has the smallest knowledge utility. That result indicates that, in the scientific crowdsourcing knowledge flow process, improving nonmonetary gain, such as knowledge utility in this paper, will eventually increase profit to the crowdsourcing solver when competing with others. That way, besides monetary incentives, crowdsourcing solvers should also pay more attention on nonmonetary incentives, such as learning more knowledge, developing new skills, etc., when participating in scientific crowdsourcing. Those nonmonetary elements are eventually important for the crowdsourcing solvers to gain high profits.

In terms of knowledge transfer cost versus solver profit, in our simulation analyses, it was assumed that $g_{A}$ was equal to $g_{B}$, and the basic unit transfer $\operatorname{cost} c=1.0$.

The simulation results are shown in Figure 3, with two scenarios to calculate solver A's profit:

1. Assume solver $B^{\prime}$ s unit cost coefficient $\mu_{B}$ equals 1 . The solver $A^{\prime}$ 's profit will change from $4 / 9$ to $1 / 2$, which is about a $+12.5 \%$ profit increase when solver $A^{\prime}$ s unit cost coefficient changes from 0 to 1 .

2. When fixing solver $A^{\prime}$ s unit cost coefficient $\mu_{A}$ to 1 and changing solver $B^{\prime}$ s unit cost coefficient $\mu_{B}$ from 0 to 1 , solver $A^{\prime}$ s profit will change from $1 / 9$ to $1 / 2$, which is a $450 \%$ profit increase.

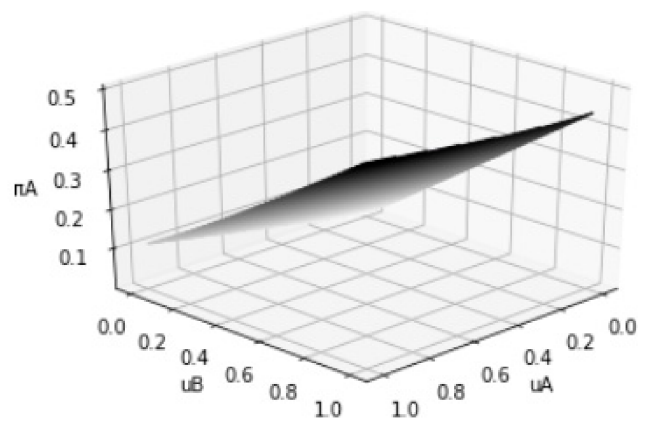

(a)

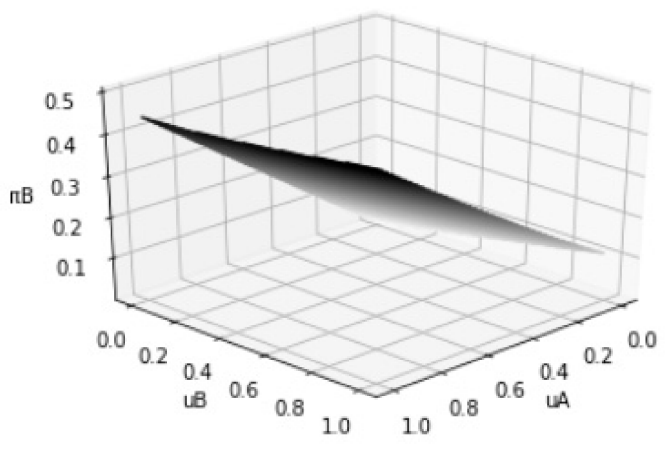

(b)

Figure 3. (a) Solver- $A$ 's profit versus knowledge utility; (b) solver- $B$ 's profit versus knowledge utility.

From crowdsourcing solver $A$ 's perspective, scenario 2 gets much more profit gain than scenario 1 . On the one hand, solver $i^{\prime}$ s profit would increase a little when $i$ 's unit transfer cost increases. This can be explained by the fact that, as unit transfer cost increases, the knowledge spill-over would increase more compared with the decreasing demand, so that profit would increase a little bit eventually. On the other hand, solver $i$ 's profit is more impacted by solver $j$ 's unit transfer cost, not solver $i$ 's own unit transfer cost. This is an interesting result, as in a knowledge flow process, a crowdsourcing solver should be a good cost controller, not because of the impact to his/her own profit, but because a higher cost would provide more profit potential to his/her competitor in the same field.

\subsection{Knowledge Distance}

In this section, a simulation of profit impacted by knowledge distance between initiator and solver is presented. We assumed a knowledge transfer $\operatorname{cost} c=1$, and both solvers had same knowledge utility, which means $g_{A}=g_{B}$. In order to examine the impact of each solver's knowledge distance to the initiator, it was necessary to fix the other solver's knowledge distance during the simulation. For solver $A^{\prime}$ s profit simulation, it was assumed that solver $B^{\prime}$ s knowledge distance $p_{B}=1$, and when simulating solver $B^{\prime}$ s profit, it was assumed that solver $A^{\prime}$ 's knowledge distance $p_{A}=1$. The simulation result are shown in Figure 4.

In the model, solver $A$ is located on left side of initiator in knowledge space and solver $B$ is on right side. The simulation result indicates that both solvers should move closer to initiator, which would 
mean the knowledge distance becomes smaller between the solver and initiator, such that more profit would be gained.

Each solver's knowledge distance has more practical implications, as the dynamic characterizations of knowledge learning when participating crowdsourcing activity, or even when preparing for crowdsourcing before real activity starts. In order to win a crowdsourcing task and gain more profit, solvers should pay attention to analysing the initiator's knowledge structure, knowledge type, knowledge terminology, etc. This effort will bring about a shorter knowledge distance and a closer relationship between the crowdsourcing initiator and solver.

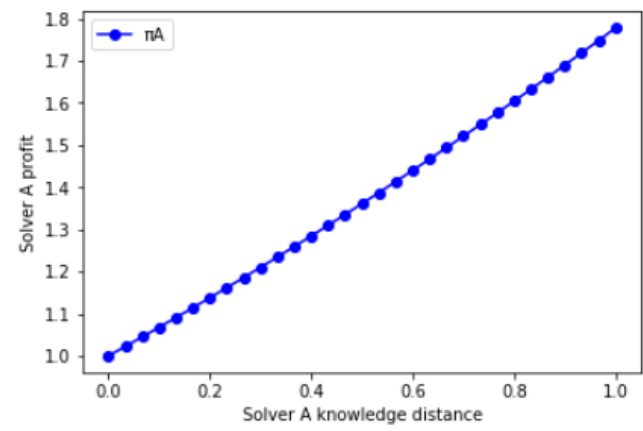

(a)

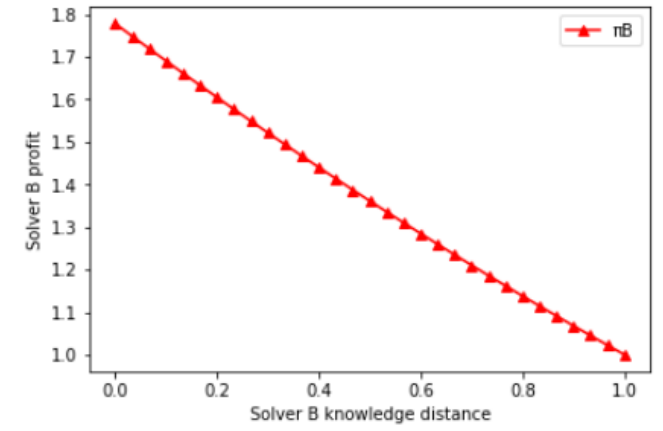

(b)

Figure 4. (a) Solver- $A$ 's profit versus knowledge distance; (b) solver- $B$ 's profit versus knowledge distance.

\subsection{Knowledge Trading Cost}

As technology and the Internet development, in order to collaborate on scientific research to solve problems, it is more common to see worldwide participants join in on scientific crowdsourcing activities; the local initiator finds a path to leverage to equilibrium. Based on Equations (26) and (27), in order to consider trading cost $t$ in game model, let us assume the knowledge transfer $\operatorname{cost} c=1$, and delta-g, which is $g_{A}-g_{B}$, means knowledge utility gap between initiator A (local participant) and initiator B (foreign participant).

In order to assess the knowledge trading cost effect, the simulation was performed with knowledge utility gap delta-g, trading cost $t$, and profit $\pi$. The result are shown in Figure 5.

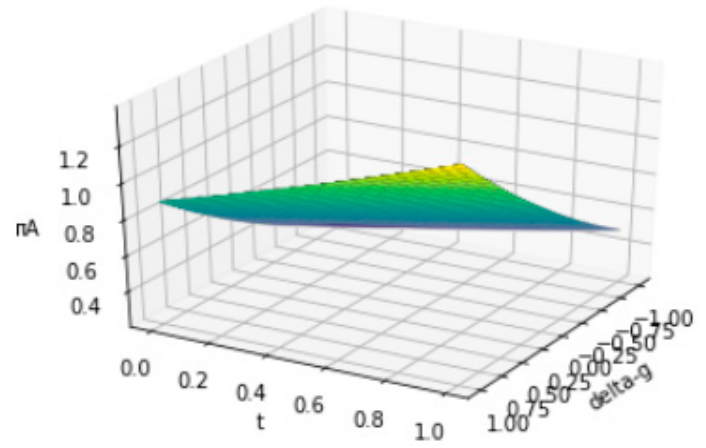

(a)

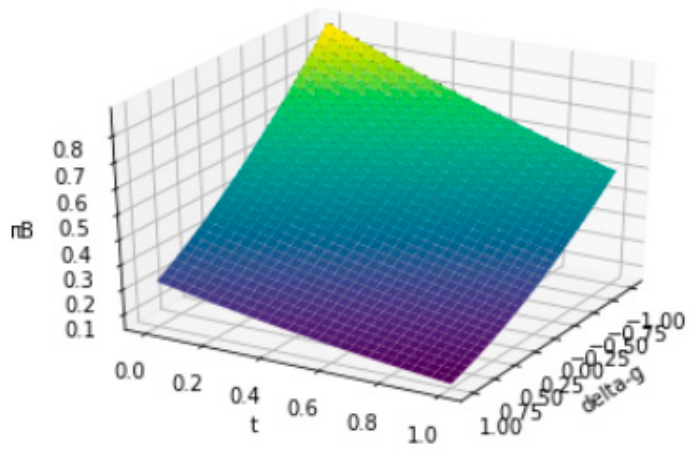

(b)

Figure 5. (a) Solver- $A$ 's profit versus trading cost; (b) solver- $B$ 's profit versus trading cost.

From the simulation result, knowledge trading cost has a significant impact on the profit of both solvers. Considering the same knowledge utility between local solver $A$ and foreign solver $B$, when trading cost increases, the local solver's profit increases about $78 \%$ and the foreign solver's profit decreases about $55 \%$. The knowledge trading cost, which is usually in the form of tax across countries, plays a critical role in leveraging the profits between a local solver and foreign solver. 
Although a local crowdsourcing solver would have a natural trading cost advantage when competing with foreign solver, effort should still be made to increase knowledge utility, since even when there is positive trading cost, a foreign solver could still have higher profit than a local solver if the gap of knowledge utility is big enough.

\section{Conclusions}

This paper investigates the solver's equilibrium when participating in a scientific crowdsourcing knowledge flow process, and employs a game model to simulate two crowdsourcing solvers' knowledge transferring and equilibrium to gain maximum profit. By studying knowledge utility, knowledge transfer cost, knowledge distance, and trading cost, the research findings provide important managerial implications for crowdsourcing solvers and initiators:

1. Crowdsourcing solvers should pay more attention to nonmonetary elements when participating in scientific crowdsourcing, such as acquiring more knowledge, developing new skills, accumulating experience, etc. By improving those nonmonetary elements, solvers should strengthen competitiveness to win crowdsourcing tasks and gain high profits eventually.

2. A crowdsourcing initiator tends to select the solver who has good cost control in the knowledge flow process. The initiator tries to use a transparent crowdsourcing platform to make it easier to select a solver with lower knowledge transfer cost, so that the relationship between initiator and solver is in active iteration game mode. This will provide fewer opportunities to solvers with higher transfer knowledge costs to gain higher profits.

3. Crowdsourcing solvers should try their best to shorten knowledge distances to initiators. It is better for crowdsourcing solvers to investigate an initiator's knowledge structure, knowledge type, knowledge terminology, etc., before starting to participate in the scientific crowdsourcing knowledge flow process.

4. The knowledge trading cost generated during scientific crowdsourcing will have impact on a solver's competitiveness. Due to a lower knowledge trading cost, a local crowdsourcing solver has natural advantage compared with a foreign crowdsourcing solver. However, knowledge trading cost is not omnipotent for competition. In order to keep the advantage, the local solver still needs make the effort to keep improving knowledge utility, or at least shorten the gap of knowledge utility compared to the foreign solver.

Although the conclusions obtained by using the game model in this research present important practical guidance for crowdsourcing solvers, we are fully aware that our study is not free of its limitations. For instance, the game model in this paper is a kind of static model for solvers; it is not suitable for dynamic situations, such as the cases where a solver's knowledge utility is changed, or the knowledge distance between solver and initiator is changed. It is very interesting to employ a dynamic game model to analyse the equilibriums of solvers in scientific crowdsourcing. Furthermore, if there are two or more initiators, from the crowdsourcing solver point of view, what are the criteria to make judgements to select the best initiator, and how should one engage in the cooperation? These are all very interesting research topics and are worth being further studied in the future.

Author Contributions: The main activities of the team of authors can be described as follows. L.Y. designed the didactic scenario, initiated the study and was significantly involved in its conception. G.W. wrote the draft of the article, designed and refined the study from a didactic and motivational perspective. Conceptualization, G.W. and L.Y.; investigation, G.W.; resources, G.W. and L.Y.; writing-original draft preparation, G.W.; writing-review and editing, L.Y.; supervision, L.Y.; project administration, L.Y.; funding acquisition, L.Y.

Funding: This research was financed by the fund provided by the General Program of National Natural Science Foundation of China, "Research on Random Symmetrical Cone Complementarity Problems and Related Topics" (number 11671250).

Acknowledgments: The authors would like to thank the reviewers for their careful reading and for providing some pertinent suggestions.

Conflicts of Interest: The authors declare no conflicts of interest. 


\section{References}

1. Howe, J. The rise of crowdsourcing. Wired Mag. 2006, 14, 1-4.

2. Oomen, J.; Aroyo, L. Crowdsourcing in the Cultural Heritage Domain: Opportunities and Challenges. In Proceedings of the 5th International Conference on Communities and Technologies, Brisbane, QLD, Australia, 29 June-2 July 2011.

3. Bayus, B. Crowdsourcing new product ideas over time: An analysis of the Dell Ideastorm community. Manag. Sci. 2013, 59, 226-244. [CrossRef]

4. Afuah, A.; Tucci, C.L. Crowdsourcing as a solution to distant search. Acad. Manag. Rev. 2012, 37, 355-375.

5. Frigerio, S.; Schenato, L.; Bossi, G.; Mantovani, M.; Marcato, G.; Pasuto, A. Hands-on Experience of Crowdsourcing for Flood Risks. An Android Mobile Application Tested in Frederikssund, Denmark. Int. J. Environ. Res. Public Health 2018, 15, 1926. [CrossRef]

6. Chiu, C.M.; Liang, T.P.; Turban, E. What Can Crowdsourcing Do for Decision Support? Decis. Support Syst. 2014, 65, 40-49. [CrossRef]

7. Seltzer, E.; Mahmoudi, D. Citizen Participation, Open Innovation, and Crowdsourcing: Challenges and Opportunities for Planning. J. Plan. Lit. 2012, 28, 3-18. [CrossRef]

8. Chesbrough, H.W. Business model innovation: Opportunities and barriers. Long Range Plan. 2010, 43, 354-363. [CrossRef]

9. Argote, L.; Miron-Spektor, E. Organizational learning: From experience to knowledge. Organ. Sci. 2011, 22, 1123-1137. [CrossRef]

10. Feller, J.; Finnegan, P.; Hayes, J.; O'Reilly, P. Orchestrating sustainable crowdsourcing: A characterization of solver brokerages. J. Strateg. Inf. Syst. 2012, 21, 216-232. [CrossRef]

11. Majchrzak, A.; Malhotra, A. Towards an Information Systems Perspective and Research Agenda on Crowdsourcing for Innovation. J. Strateg. Inf. Syst. 2013, 22, 257-268. [CrossRef]

12. Piazza, M.; Mazzola, E.; Acur, N.; Perrone, G. Governance Considerations for Seeker-Solver Relationships: A Knowledge-Based Perspective in Crowdsourcing for Innovation Contests. Br. J. Manag. 2019, 1-19. [CrossRef]

13. Garcia Martinez, M. Solver engagement in knowledge sharing in crowdsourcing communities: Exploring the link to creativity. Res. Policy 2015, 44, 1419-1430. [CrossRef]

14. Xu, Z.; Zhang, H.; Hu, C.; Mei, L.; Xuan, J.; Choo, K.-K.R.; Zhu, Y. Building knowledge base of urban emergency events based on crowdsourcing of social media. Concurr. Comput. Pract. Exp. 2016, 28, 4038-4052. [CrossRef]

15. Jiang, Z.S.; Hu, L.Y. Analysis on knowledge transfer decisions in technology innovation alliance based on leader-Followers games. Sci. Res. Manag. 2011, 32, 19-25.

16. Litman, L.; Robinson, J.; Abberbock, T. TurkPrime.com: A versatile crowdsourcing data acquisition platform for the behavioural sciences. Behav. Res. Methods 2016, 49, 433-442. [CrossRef]

17. Andrius, A.; Raimonda, A. Value Creation in Innovations Crowdsourcing: Example of Creative Agencies. Organ. Mark. Emerg. Econ. 2013, 2, 72-103.

18. Arolas, E.; de Guevara, F. Towards an integrated crowdsourcing definition. J. Inf. Sci. 2012, 38, 189-200. [CrossRef]

19. Liu, X.; Giroud, A. International knowledge flows in the context of emerging-economy MNEs and increasing global mobility. Int. Bus. Rev. 2016, 25, 125-129. [CrossRef]

20. Grant, R.M.; Baden-Fuller, C. A knowledge accessing theory of strategic alliances. J. Manag. Stud. 2004, 41, 61-84. [CrossRef]

21. Liu, Y.; Deng, P.; Wei, J.; Ying, Y.; Tian, M. International R\&D alliances and innovation for emerging market multinationals: Roles of environmental turbulence and knowledge transfer. J. Bus. Ind. Mark. 2019. [CrossRef]

22. Battistella, C.; Nonino, F. Open Innovation Web-based Platforms: The Impact of Different Forms of Motivation on Collaboration. Innov. Manag. Policy Pract. 2012, 14, 557-575. [CrossRef]

23. Malhotra, A.; Majchrzak, A.; Kesebi, L.; Looram, S. Developing innovative solutions through internal crowdsourcing. MIT Sloan Manag. Rev. 2017, 58, 73-79.

24. Wang, G.H.; Yu, L.Y. Differential Game Analysis of Scientific Crowdsourcing on Knowledge Transfer. Sustainability 2019, 11, 2735. [CrossRef] 
25. Fang, M.; Yin, J. Active learning for crowdsourcing using knowledge transfer. In Proceedings of the Twenty-Eighth AAAI Conference on Artificial Intelligence, Québec City, QC, Canada, 27-31 July 2014; pp. 1809-1815.

26. Xue, J.; Ding, C.Q.; Yuan, J. The evolutionary game study of knowledge transfer behaviour in cooperative crowdsourcing community of innovation. Adv. Model. Anal. A 2016, 53, 164-175.

27. Albors, J.; Ramos, J.; Hervas, J. New learning network paradigms: Communities of objectives, crowdsourcing, wikis and open source. Int. J. Inf. Manag. 2008, 28, 194-202. [CrossRef]

28. King, A.A.; Lakhani, K.R. The contingent effect of absorptive capacity: An open innovation analysis. Harv. Bus. Sch. Work. Pap. Ser. 2011, 21, 11-102. [CrossRef]

29. Patrick, P.; Lüttgens, D.; Piller, F.T. How firms develop capabilities for crowdsourcing to increase open innovation performance: The interplay between organizational roles and knowledge processes. J. Prod. Innov. Manag. 2018. [CrossRef]

30. Rosell, D.T.; Lakemond, N.; Wasti, S.N. Integrating knowledge with suppliers at the R\&D-manufacturing interface. J. Manuf. Technol. Manag. 2014, 25, 240-257.

31. Naidorf, J.C. Knowledge utility: From social relevance to knowledge mobilization. Educ. Policy Anal. Arch. 2014, 22, 1-31.

32. Battistella, C.; de Toni, A.F.; Pillon, R. Inter-organisational technology/knowledge transfer: A framework from critical literature review. J. Technol. Transf. 2016, 41, 1195-1234. [CrossRef]

33. Krylova, K.O.; Vera, D.; Crossan, M. Knowledge transfer in knowledge-intensive organizations: The crucial role of improvisation in transferring and protecting knowledge. J. Knowl. Manag. 2016, 20, 1045-1064. [CrossRef]

34. Winkelbach, A.; Walter, A. Complex technological knowledge and value creation in science-to-industry technology transfer projects: The moderating effect of absorptive capacity. Ind. Mark. Manag. 2015, 47, 98-108. [CrossRef]

35. Chen, B. Distance of knowledge and pricing knowledge. Stud. Sci. Sci. 2007, 1, 14-18.

36. Capaldo, A.; Lavie, D.; Messeni Petruzzelli, A. Knowledge Maturity and the Scientific Value of Innovations. J. Manag. 2016, 43, 503-533. [CrossRef]

37. Galati, F.; Bigliardi, B. Redesigning the model of the initiative and evolution of inter-firm knowledge transfer in R\&D relationships. J. Knowl. Manag. 2019. [CrossRef]

38. Brabham, D.C. Crowdsourcing the public participation process for planning projects. Plan. Theory 2009, 8, 242-262. [CrossRef]

39. Marjanovic, S.; Fry, C.; Chataway, J. Crowdsourcing based business models: In search of evidence for innovation 2.0. Sci. Public Policy 2012, 39, 318-332. [CrossRef]

40. Cai, W.Y.; Zhang, J.Y. Analysis of Knowledge Spillover and Technological Progress in Globalization. Jianghuai Forum 2009, 6, 47-53.

41. Jiang, X.; Bao, Y.; Xie, Y.; Gao, S. Partner trustworthiness, knowledge flow in strategic alliances, and firm competitiveness: A contingency perspective. J. Bus. Res. 2016, 69, 804-814. [CrossRef]

42. Eriksson, K.; Johanson, J.; Majkgård, A.; Sharma, D.D. Experiential Knowledge and Cost in the Internationalization Process. Knowl. Netw. Power 2015, 28, 337-360.

43. Cassiman, B.; Valentini, G. Open innovation: Are inbound and outbound knowledge flows really complementary? Strateg. Manag. J. 2015, 37, 1034-1046. [CrossRef]

44. De Frutos, M.A.; Hamoudi, H. Spatial competition with concave transport costs. Reg. Sci. Urban Econ. 2002, 32, 531-540. [CrossRef]

45. Brenner, S.H. Hotelling games with three, four, and more players. J. Reg. Sci. 2005, 45, 851-864. [CrossRef]

46. Sengupta, A.; Sengupta, K. A Hotelling-Downs mode 1 of electoral competition with the option to quit. Games Econ. Behav. 2008, 62, 661-674. [CrossRef]

(C) 2019 by the authors. Licensee MDPI, Basel, Switzerland. This article is an open access article distributed under the terms and conditions of the Creative Commons Attribution (CC BY) license (http://creativecommons.org/licenses/by/4.0/). 